\title{
Preparation of monoclonal antibody based indirect competitive ELISA for detecting 19-nortestosterone residue
}

\author{
JIANG JinQing ${ }^{1,2}$, ZHANG HaiTang ${ }^{1}$, FAN GuoYing ${ }^{1}$, MA JinYou ${ }^{1}$, WANG ZiLiang $^{1 *}$ \& \\ WANG JianHua ${ }^{2}$ \\ ${ }^{1}$ College of Animal Sciences, Henan Institute of Science and Technology, Xinxiang 453003, China; \\ ${ }^{2}$ College of Veterinary Medicine, Northwest A\& F University, Yangling 712100, China
}

Received December 13, 2010; accepted April 19, 2011

\begin{abstract}
19-Nortestosterone (NT) has been illegally used in horse racing to boost physical performance, and in animal husbandry to accelerate weight gain. To monitor the abuse of NT, our goal was to develop a commercial enzyme linked immunosorbent assay (ELISA) kit. For this purpose, hybridomas were prepared by fusing NS0 mouse myeloma cells with splenocytes isolated from immunized BALB/c mouse. Noncompetitive and competitive indirect ELISA were used to screen positive cell clones. To optimize the indirect competitive ELISA (icELISA) method, various methanol concentrations in assay buffer were evaluated. Matrix effects in urine and spiking test were also investigated. Finally, five hybridoma cell lines named NT-1, NT-2, NT-3, NT-4 and NT-5 were screened out. The corresponding monoclonal antibodies (mAbs) were of the $\mathrm{IgG}_{1}$ isotype with a $k$ light chain, and the antibody affinity of all mAbs were between $2.6 \times 10^{9}$ and $4.7 \times 10^{9} \mathrm{~L} / \mathrm{mol}$. The titer and $\mathrm{IC}_{50}$ values of purified ascites were in the range of $0.64 \times 10^{5}-2.56 \times 10^{5}$ and $0.55-1.0 \mathrm{ng} / \mathrm{mL}$, respectively. Based on the NT-1 hybridoma, a heterologous icELISA method was developed for the quantitative detection of NT in cattle urine. The dynamic range was from 0.004 to $85.8 \mathrm{ng} / \mathrm{mL}$, with a detection limit for the assay and $\mathrm{IC}_{50}$ values of 0.002 and $0.55 \mathrm{ng} / \mathrm{mL}$, respectively. Except for a high cross-reactivity (62\%) to $\alpha-\mathrm{NT}$, negligible cross-reactivity to other compounds was observed. After optimization, $10 \%$ of methanol was used in the assay buffer, and a 20-fold dilution in cattle urine gave an inhibition curve almost the same as that in phosphate buffered saline. The correlation coefficient between the established icELISA and LC-MS/MS method was 0.9871. The results showed that the established heterologous icELISA method provides an excellent alternative for the detection of NT residues in food producing animals.
\end{abstract}

19-nortestosterone, hybridoma cell lines, monoclonal antibody, indirect competitive ELISA, urine

Citation: Jiang J Q, Zhang H T, Fan G Y, et al. Preparation of monoclonal antibody based indirect competitive ELISA for detecting 19-nortestosterone residue. Chinese Sci Bull, 2011, 56: 2698-2705, doi: 10.1007/s11434-011-4604-y

19-Nortestosterone (17 $\beta$-hydroxyestr-4-en-3-one, NT), also named nandrolone, is a xenobiotic with androgenic and anabolic properties known since the 1930s. Recently, it has been found endogenously in a number of species [1]. In the 1950 s, the recognition of the growth promoting property of NT led to it being used to accelerate meat production. It can enhance lean tissue growth, reduce fat deposition, increase the weight gain and feed conversion efficiency of animals [2-4]. Administration is usually by intramuscular injection of the esterified drug in suspension with benzyl alcohol and

*Corresponding author (email: wangzl_2008@yahoo.com.cn) arachis oil, or inserted into the fleshy part of the ear. The hormone is gradually released over a period of $50 \mathrm{~d}$ or so, to ensure a relatively constant and slightly elevated level in the animal's blood [5]. Because of its androgenic activity, NT has also been employed as a doping agent for athletes and in horse racing to increase muscle mass and physical strength, and boost physical performance $[4,6]$.

But studies show NT and its metabolite residue in meat produce a series of adverse effects, including peliosis hepatitis, hypoproteinemia, adrenal atrophy, dyskeratosis, reduced semen production, testicle shrinkage, menstrual cycle disorder, masculinization, cerebral dysfunction, emotional 
instability and other side effects [7-10]. Consequently the use of natural and synthetic hormones for growth promotion purposes in meat-producing animals has been prohibited in the European Community since 1986 to protect consumers from possible developmental, neurobiological, genotoxic and carcinogenic effects [11]. NT and its esters, as well as metabolites are also prohibited in animal food and animal drugs and is on the banned compounds list in China (Notice No. 193 of the Ministry of Agriculture in April 2002). However, illegal use of NT as a growth promoter has been widely reported throughout Europe [2] and in China, thus prompting continuous surveillance to control its abuse.

Traditionally, NT residue analysis has relied upon classical analytical methods, such as gas chromatography coupled to mass spectrometric (GC-MS) [6,12-14], liquid chromatography coupled to mass spectrometric (LC-MS) [15-17], and other quantitative methods. Chromatographic techniques generally require highly skilled personnel, laborious sample pretreatment and high-cost, complex equipment. Therefore, they are not suitable for routine analysis of a large number of samples or on-site determinations. Immunological techniques are increasingly considered as alternative and/or complementary methods for residue analysis because of their simplicity, cost-effectiveness, portability, and high sample throughput.

Our main goal was to produce high-sensitivity monoclonal antibodies displaying excellent affinity and specificity for NT, and optimize the indirect competitive ELISA protocols based on selected monoclonal antibodies. This work potentially optimizes the pre-treatment procedures for LC-MS and GC-MS detection, and lays a solid foundation for NT-kit and test strip development.

\section{Materials and methods}

\subsection{Materials and chemicals}

19-Nortestosterone (NT), 17a, 19-nortestosterone (a-NT), estradiol, trenbolone, methyltestosterone and clostebol were purchased from Dr. Ehrenstorfer Company (Germany). NT-17-succinic anhydride ester-BSA (NT-17-BSA) as immunogen and NT-3-carboxymethyloxime-OVA (NT-3-OVA) as coating antigen were conjugated in our laboratory. Freund's complete adjuvant and Freund's incomplete adjuvant were obtained from Pierce. GaMIgG-HRP (whole molecule specific) was purchased from Sino-American Biotechnology Company (Shanghai, China). Transparent 96well polystyrene microtiter plates (Boyang Experimental Equipment Factory, Jiangsu, China) were used for the colorimetric measurement. Hypoxanthine/thymidine/aminopterin and hypoxanthine/thymidine were obtained from SigmaAldrich (USA). RPMI-1640 with $L$-glutamine was obtained from Gibco. Polyethylene glycol 1500 (PEG 1500, 50\%) was from Roche Diagnostics Corporation (Indianapolis,
USA). Fetal bovine serum was from Hangzhou Sijiqing Biological Engineering Materials Co. Ltd. (Hangzhou, China). Cell culture plates (24 and 96 wells) and culture flasks were obtained from Costar Inc. (Bethesda, MD, USA). A mouse monoclonal antibody isotyping kit was purchased from Pierce Biotechnology, Inc. (Rockford, II, USA). 3,3,5,5-Tetramethylbenzidine (TMB), phenacetin, urea peroxide were obtained from Sigma Company. All other solvents and reagents were of analytical grade or higher, unless otherwise stated.

Eight-week-old female BALB/c mice were obtained from the Laboratory Animal Center, Beijing Medical University, China, and raised under strictly controlled conditions in our laboratory.

\subsection{Buffers}

The buffers used were: phosphate buffered saline (PBS), consisting of $\mathrm{NaCl}(137 \mathrm{mmol} / \mathrm{L}), \mathrm{Na}_{2} \mathrm{HPO}_{4} \cdot 12 \mathrm{H}_{2} \mathrm{O}(10$ $\mathrm{mmol} / \mathrm{L}), \mathrm{KCl}(2.68 \mathrm{mmol} / \mathrm{L})$ and $\mathrm{KH}_{2} \mathrm{PO}_{4}(1.47 \mathrm{mmol} / \mathrm{L})$, $\mathrm{pH}$ 7.4; carbonate buffered saline (CBS) consisted of $\mathrm{Na}_{2} \mathrm{CO}_{3}$ (15 mmol/L), $\mathrm{NaHCO}_{3}$ (35 mmol/L), pH 9.6; washing buffer consisted of PBS containing $0.05 \%$ Tween20 ; blocking buffer consisted of BSA $(1 \%$, w/v) in PBS. Substrate buffer consisted of adding part A $(500 \mathrm{~mL})$ and part B $(500 \mathrm{~mL})$ solutions together. Part A contained (per $1 \mathrm{~L}$ of water) $3.15 \mathrm{~g}$ of citric acid, $6.966 \mathrm{~g}$ of anhydrous sodium acetate, $0.08 \mathrm{~g}$ of phenacetin and $0.05 \mathrm{~g}$ of urea peroxide adjusted to $\mathrm{pH} 5$ with $\mathrm{HCl}$. Part B contained $1.27 \mathrm{~g}$ TMB dissolved in $500 \mathrm{~mL}$ of methanol and $500 \mathrm{~mL}$ of glycerol. The stopping solution was $2 \mathrm{~mol} / \mathrm{L} \mathrm{H}_{2} \mathrm{SO}_{4}$.

\subsection{Instruments}

A spectrophotometric microtiter reader (MULTISKAN MK3, Thermo Company, USA), provided with a $450 \mathrm{~nm}$ filter, was used for absorbance measurements. Transferpettors (eLINE, Proline) were obtained from Biohit Company (Finland). A Legend Micro 17 microcentrifuge and GS15R high-speed refrigerated centrifuge were supplied by Thermo Company (USA). $\mathrm{A} \mathrm{CO}_{2}$ incubator from RS-Biotech (Galaxy S+, UK) was used for cell cultivation. A SW-CJ-2FD Superclean Bench was purchased from Suzhou Purification Equipment Co., Ltd (Suzhou, China). An inverted microscope (TS100F, Nikon Company, Japan) was used for cell observation. DK-8D thermostatic bathes were from Yiheng Instruments Co., Ltd (Shanghai, China). BS124S electronic balance was from the Sartorius Group (Germany). LDZX-30KB Steam Sterilizer was supplied by Shenan Medical Instrument Factory (Shanghai, China). Deionized water was prepared using an Ultra class UV plus water purification system (SG Company, Germany). Incubation for immunoreactions was carried out in a DH-360A oven from Zhongxingweiye Instrument Co., Ltd (Beijing, China). 


\subsection{General ELISA procedure}

The titer of the antibody was tested by indirect ELISA, using the procedure described below. The microplates were coated with coating antigen NT-3-OVA at $2 \mu \mathrm{g} / \mathrm{mL}$ (100 $\mu \mathrm{L} /$ well) by overnight incubation at $4^{\circ} \mathrm{C}$. Plates were washed with PBST three times and unbound active sites were blocked with $250 \mu \mathrm{L} /$ well of blocking buffer, followed by incubation for $2 \mathrm{~h}$ at room temperature. The solution was then discarded, and plates were washed three times with washing solution. Fifty $\mu \mathrm{L} /$ well of appropriate dilution of the antisera was added, and the plates were incubated for 15 $\min$ at $37^{\circ} \mathrm{C}$. After another washing procedure, GaMIgG-HRP $(1: 1000,50 \mu \mathrm{L} / w e l l)$ was added, followed by incubation for $25 \mathrm{~min}$ at $37^{\circ} \mathrm{C}$. The plates were washed, and $60 \mu \mathrm{L} /$ well freshly prepared substrate solution A and B $(1: 1, \mathrm{v} / \mathrm{v})$ was added. After incubating at room temperature for $15 \mathrm{~min}$, the reaction was stopped using $2 \mathrm{~mol} / \mathrm{L} \mathrm{H}_{2} \mathrm{SO}_{4}$. The absorbance was measured at $450 \mathrm{~nm}$ and the antibody titer was defined as the reciprocal of the dilution that resulted in an absorbance value that was twice that of the background. Absorbances were corrected by blank readings (wells in which no primary antibody was added).

Indirect competitive ELISA (icELISA) was employed to determine the sensitivity and specificity. Checkerboard tests were performed to determine optimal dilution of the coating antigen and the primary antibody, resulting in the following optimized protocol. Ninety-six well polystyrene ELISA plates were coated with $100 \mu \mathrm{L}$ per well of OVA-conjugate solution, then received $50 \mu \mathrm{L}$ per well of analyte in PBS containing $5 \%$ methanol plus $50 \mu \mathrm{L}$ per well of hybridoma supernatant or purified antibody solution in PBST, unless otherwise stated. All samples were run in triplicate. The same procedure was followed for the indirect ELISA. With the inhibition ELISA format, analytes that do not react with the antibody would produce absorbance near $100 \%$; conversely, analytes that do react with the antibody would decrease in percentage of absorbance. The inhibition rate was expressed as $\left(B / B_{0}\right) \%$, where $B$ is the absorbance of the well containing the competitor and $B_{0}$ is the absorbance of the well without the competitor. Standard curves were calculated by mathematically fitting experimental points to a fourparameter logistic equation.

\subsection{Immunization schedule}

Five BALB/c female mice (8-10 weeks old) were immunized with NT-17-BSA conjugates by subcutaneously injections at multiple points. The first dose consisted of $60 \mu \mathrm{g}$ of immunogen as an emulsion of PBS and Freund's complete adjuvant. Three subsequent injections were given at 3-week intervals with the same dosage of immunogen emulsified in Freund's incomplete adjuvant. After a resting period of at least 3 weeks from the last injection with adjuvant, mice were tail-bled and screened for anti-NT activity by icELISA.
The mouse showing the highest anti-NT activity received a final soluble intraperitoneal injection of $100 \mu \mathrm{g}$ of conjugate in PBS, 3-4 d prior to cell fusion.

\subsection{Fusion of myeloma and spleen cells}

Portions of the cell fusion procedures and cloning conditions were described previously by Köhler and Milstein [18] with further modifications by Chen et al. [19]. Briefly, NS0 myeloma cells were passed through a medium containing 8 -azaguanine and then grown for $4-5 \mathrm{~d}$ at $37^{\circ} \mathrm{C}$ in a $5 \%$ $\mathrm{CO}_{2}$ atmosphere. On the day of fusion, the mouse (that received the booster injection) was sacrificed by cervical dislocation and the spleen was removed aseptically. The splenocytes were isolated and fused with myeloma cells at a 10:1 ratio using PEG 1500 as the fusing agent, followed by gentle stirring for $1 \mathrm{~min}$. The resulting mixture was kept still at $37^{\circ} \mathrm{C}$ for $1.5 \mathrm{~min}$, and then $40 \mathrm{~mL}$ of hypoxanthine/ thymidine/aminopterin-1640 medium (supplemented with $15 \%$ fetal bovine serum) was slowly added to the fused cells. The fused cells were then distributed into 96-well culture plates, in which mouse peritoneal macrophages were prepared on the day before the fusion and were grown with the selective hypoxanthine/thymidine/aminopterin medium.

\subsection{Hybridoma production, selection, and cloning}

Ten-fourteen days after fusion, supernatants from hybridoma colonies were recovered and screened using a combination of noncompetitive and competitive, indirect ELISA. Well cultures showing significant NT recognition activity were expanded from the culture in the 96-well plate to a 24-well plate, and subcloned three times by limiting dilution. The wells picked for expansion were viewed under a microscope to confirm the presence of a single cell source to ensure their monoclonal origin, and the hypoxanthine/thymidine medium was gradually replaced by complete medium. After hybridomas became dense in the 24-well plate, they were transferred to 50 or $100 \mathrm{~mL}$ culture flasks. Hybridomas cells were collected, centrifuged and the supernatants were stored at $-20^{\circ} \mathrm{C}$ until used. Colonies of interest were frozen in culture medium containing 10\% dimethyl sulfoxide (DMSO) and cryopreserved in liquid nitrogen, followed by defrosting three times to select the stable antibody producing clones.

\subsection{Monoclonal antibody production}

A mature female BALB/c mouse was injected intraperitoneally with $0.5 \mathrm{~mL}$ of paraffin $10 \mathrm{~d}$ before receiving an intraperitoneal injection of the positive hybridoma cells suspended in RPMI 1640 medium. Ascitic fluid was collected $10 \mathrm{~d}$ after the injection and then stored at $-20^{\circ} \mathrm{C}$ until use. Purification of monoclonal antibody (mAb) was performed according to the modified caprylic acid ammonium sulfate precipitation (CAASP) method described before [20]. 


\subsection{Characterization of $\mathrm{mAbs}$}

The purified antibody was immediately used for the characterization. The protein content of the antibody was determined according to the following formula: protein concentration (mg/ $\mathrm{mL})=1.45 A_{280 \mathrm{~nm}}-1.74 A_{260 \mathrm{~nm}}$, where $A$ value is the optical density [21]. Measurement of monoclonal antibody affinity (Ka) was carried out according to the procedure described by Wang et al. [22]. The class and subclass of the isotypes of the purified antibody were determined by using a mouse monoclonal antibody isotyping kit. The calibration curves were fitted based on the average of three separate assays in triplicate.

\subsection{Establishment and optimization of icELISA}

The following values were evaluated to identify the icELISA. The $\mathrm{IC}_{50}$ values were calculated to determine the sensitivity, which represented the concentration of NT that produced $50 \%$ inhibition of antibody binding to the coating antigen. The detection limit of the assay was defined as the lowest concentration that exhibits a signal of $15 \%$ inhibition [23]. The dynamic range for the icELISA was calculated as the concentration of the analyte providing a $20 \%-80 \%$ inhibition rate $\left(\mathrm{IC}_{20}-\mathrm{IC}_{80}\right.$ values) of the maximum signal. Specificity was defined as the ability of structurally related chemicals to bind to the specific antibody [24] and crossreactivity was calculated as: (( $\mathrm{IC}_{50}$ of $\left.\mathrm{NT}\right) /\left(\mathrm{IC}_{50}\right.$ of analogs $)$ ) $\times 100 \%$. The lower the cross-reactivity is, the higher the specificity of NT mAb is.

NT stock solution was prepared in PBS containing different concentrations of methanol. To optimize the schedule, the standards were prepared using 5\%, 10\%, 20\%, 30\%, and $40 \%$ of methanol. The effects on icELISA standard curve were evaluated by $A_{\max }$ and $\mathrm{IC}_{50}$ values.

\subsection{Matrix effects in authentic urine sample}

Portions of the procedure were depicted earlier [25]. Briefly, a total of $10 \mathrm{~mL}$ of cattle urine sample was centrifuged at $4^{\circ} \mathrm{C}$ with a speed of $3000 \mathrm{r} / \mathrm{min}$ for $10 \mathrm{~min}$, and the supernatant layer was transferred into a calibrated flask. In order to reduce the background and assess matrix interference, the urine sample was diluted in PBS (total 2, 5, 10 and 20-fold dilution) before they were applied to the microtiter plate. Typical experimental response curves were plotted for the absorbance values against the different concentrations of NT. $B$ and $B_{0}$ values from each diluted curve were compared with that generated from the PBS to determine the appropriate urine dilution.

\subsection{LC-MS/MS determination}

In this study, we followed the method described by Strahm et al. [26], with significant modifications. Briefly, $10 \mathrm{~mL}$ of urine sample was centrifuged at $3500 \mathrm{r} / \mathrm{min}$ for $5 \mathrm{~min}$, and a volume of $10 \mathrm{~mL}$ acetate buffer $(\mathrm{pH} \mathrm{5.2,} 0.2 \mathrm{~mol} / \mathrm{L})$ was added into the supernatant. The mixture was then subjected to enzymatic hydrolysis with $50 \mu \mathrm{L}$ of $\beta$-glucuronidase from $E$. coli (Sigma-Aldrich, USA) and incubated on an oscillator at $37^{\circ} \mathrm{C}$ for $6 \mathrm{~h}$. The mixture was then extracted with $10 \mathrm{~mL}$ of methanol and submitted to solid-phase for the clean-up process. The $\mathrm{SPE} \mathrm{C}_{18}$ cartridges (Dalian Sipore Co., Ltd., China) were consecutively conditioned with $5 \mathrm{~mL}$ of methanol and then $5 \mathrm{~mL}$ of deionized water at a flow rate of $0.3 \mathrm{~mL} / \mathrm{min}$. After loading with the aqueous extract solution, the cartridge was washed with $10 \mathrm{~mL}$ of the elution solution ( $n$-hexane- ether $[70: 30, \mathrm{v} / \mathrm{v}])$ at a flow rate of less than 0.5 $\mathrm{mL} / \mathrm{min}$. After centrifugation, the organic layer was removed under a stream of nitrogen in a water bath at $45^{\circ} \mathrm{C}$, and the extracts were redissolved in methanol for further analysis.

The LC-MS/MS analysis were performed on a SURVEYOR liquid chromatograph (Thermo Company, USA), equipped with a PDA plus Detector (Thermo Finnigan, USA) and a Thermo ODS-HYPERSIL column $(3 \mu \mathrm{m}, 2.1 \times 150 \mathrm{~mm})$. Analytes were separated using a mobile phase solution of $1 \%$ formic acid in water/acetonitrile/methanol (60:20:20, $\mathrm{pH} 2.5$ ) at a constant temperature of $30^{\circ} \mathrm{C}$ and a flow rate of $0.2 \mathrm{~mL} / \mathrm{min}$. The ultraviolet detector was operated at $242 \mathrm{~nm}$ and the injection volume was $10 \mu \mathrm{L}$. Mass spectrometry was performed using a mass spectrometer equipped with a TurbolonSpray ESI source (LCQ Deca XP MAX, Finnigan Company, USA). Positive chemical ionization (PCI) mode was used and the relative collision energy was optimized to $28 \%$. A pseudo-molecular ion $[\mathrm{M}+\mathrm{H}]^{+}$was selected as the parent ion for fragmentation, and the corresponding fragment ions were used for identification and quantitation.

\section{Results}

\subsection{Selection for potential cell-fused mice}

Five mice were immunized with the NT-17-BSA conjugate following the standard protocols described above, while NT-3-OVA were coated onto ELISA plates to determine the titer and inhibition level of antisera. After three subsequent injections, four of the five mice immunized with NT-17BSA produced antisera with significant anti-NT activities (Figure 1). From the inhibition curves obtained, N0.4 mouse afforded the most sensitive $\mathrm{IC}_{50}$ value $(17.5 \mathrm{ng} / \mathrm{mL})$ and it was selected for further use.

\subsection{Production of monoclonal antibodies}

Ten days following the fusion, growing hybridoma cell clones could be observed in many wells of the seeded 96well plates. The fusion rate of the mouse spleen cells with myeloma cells was about $85 \%$. Supernatants of all wells were screened by simultaneous noncompetitive and competitive assays, and the positive well rate was $18 \%$. Selection 


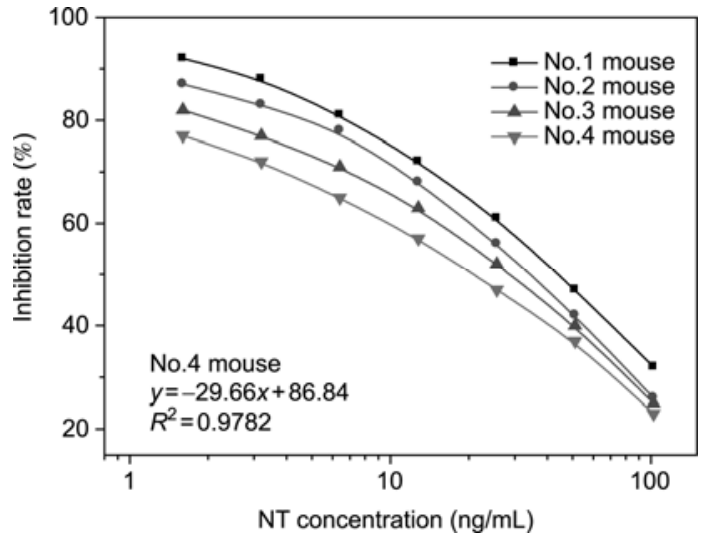

Figure 1 Inhibitive curves of NT antisera against NT by indirect competitive ELISA.

of clones from these positive cultures by limiting dilution led to five stable hybridoma cell lines. These monoclonal cultures and their corresponding cell lines were named NT-1, NT-2, NT-3, NT-4 and NT-5. Using a mouse monoclonal antibody isotyping kit, all five antibodies were of the $\mathrm{IgG}_{1}$ isotype with $k$ light chain. The protein concentrations of all $\mathrm{mAbs}$ were between 5.6 and $8.4 \mathrm{mg} / \mathrm{mL}$. Finally, five hybridomas were expanded and stored in liquid nitrogen. The mAbs from culture supernatants and ascites were purified and characterized. Based on the results of the checkerboard titration, the antibody titers and $\mathrm{IC}_{50}$ values were determined. The results are shown in Table 1. From the inhibition curves obtained, the most sensitive hybridomas, named NT- 1 and NT-2, showed the $\mathrm{IC}_{50}$ values of 0.55 and $0.58 \mathrm{ng} / \mathrm{mL}$, respectively. This means that the sensitivity of $\mathrm{mAb}$ increased approximately 30 fold in comparison to that of the NT pAb tested before. Therefore, NT-1 hybridoma was selected for further evaluation of mAb specificity and subsequent immunoassay development.

\subsection{Hybridoma stability experiments}

Five hybridoma cell lines that produced the antibodies of interest were identified by stability verification. After three times of frozen and defrosted procedure, the performances of NT-1 and NT-2 hybridomas were more consistent in the experiments. The results are shown in Figure 2.

\subsection{Determination for the affinity constant (Ka) of NT mAbs}

The affinity constant produced by each of the five selected hybridomas was measured by ELISA using serial dilutions of both coated antigen and NT mAb. The optimal condition of ELISA in which the $A$ value was about 1.0 was selected. The apparent $K$ as of NT-1, NT-2, NT-3, NT-4 and NT-5 $\mathrm{mAb}$ were $4.7 \times 10^{9}, 4.2 \times 10^{9}, 3.9 \times 10^{9}, 2.9 \times 10^{9}$ and $2.6 \times 10^{9}$ $\mathrm{L} / \mathrm{mol}$, respectively (Figure 3 ).
Table 1 Titers and $\mathrm{IC}_{50}$ results of mAbs produced by five hybridomas

\begin{tabular}{cccc}
\hline $\begin{array}{c}\text { Monoclonal } \\
\text { antibody }\end{array}$ & $\begin{array}{c}\text { Titer of culture } \\
\text { supernatants }\end{array}$ & $\begin{array}{c}\text { Titer of } \\
\text { ascites }\end{array}$ & $\begin{array}{c}\mathrm{IC}_{50} \text { values of } \\
\text { ascites }(\mathrm{ng} / \mathrm{mL})\end{array}$ \\
\hline NT-1 & $5.12 \times 10^{2}$ & $2.56 \times 10^{5}$ & 0.55 \\
NT-2 & $5.12 \times 10^{2}$ & $2.56 \times 10^{5}$ & 0.58 \\
NT-3 & $2.56 \times 10^{2}$ & $1.28 \times 10^{5}$ & 0.8 \\
NT-4 & $1.28 \times 10^{2}$ & $1.28 \times 10^{5}$ & 0.93 \\
NT-5 & $1.28 \times 10^{2}$ & $0.64 \times 10^{5}$ & 1.0 \\
\hline
\end{tabular}

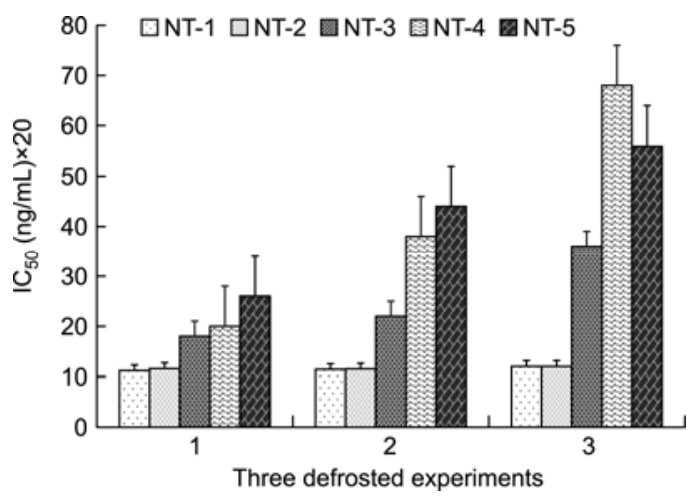

Figure $2 \mathrm{IC}_{50}$ values of NT mAb generated from five selected hybridomas after three defrosted times.

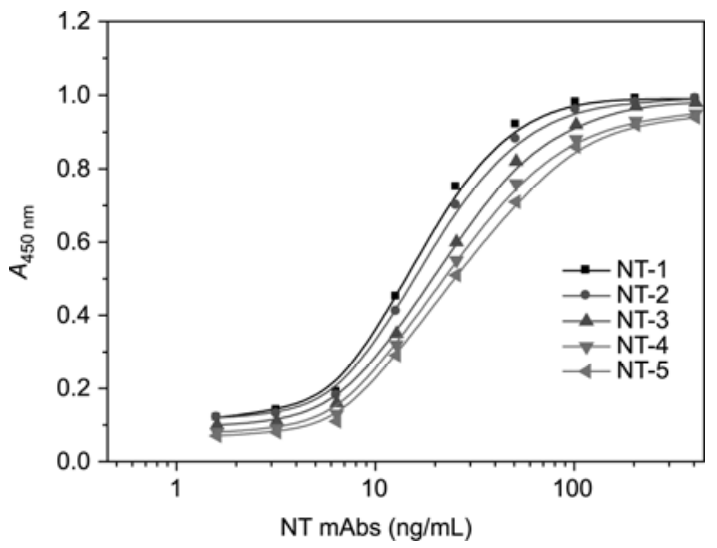

Figure 3 Affinity constant $(K a)$ curves of NT mAbs.

\subsection{Establishment of heterologous icELISA standard curve}

From the checkerboard titration (data not shown), a representative standard inhibition curve is shown in Figure 4. In this assay, NT-17-BSA was employed as the immunogen and NT-3-OVA was used as the coating antigen to pursue the heterologous sensitivity. Based on the results, the optimum concentration of coating antigen was $2 \mu \mathrm{g} / \mathrm{mL}$ and $\mathrm{mAb}$ was 1:50000 dilutions. This assay allowed the detection of NT (20\%-80\% inhibition of color development) from 0.004 to $85.8 \mathrm{ng} / \mathrm{mL}$, with an $\mathrm{IC}_{50}$ value of $0.55 \mathrm{ng} / \mathrm{mL}$. The detection limit of the assay was $0.002 \mathrm{ng} / \mathrm{mL}$. 


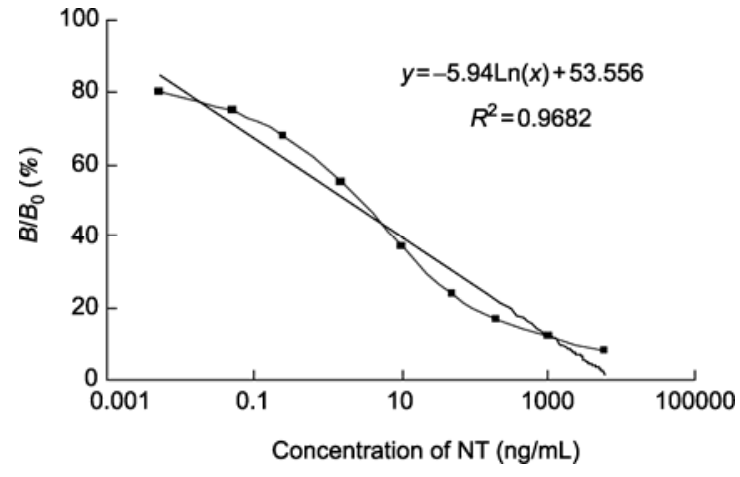

Figure 4 Optimized standard heterologous icELISA inhibition curve for NT. Data were obtained by averaging three independent curves, each run in triplicate. NT-3-OVA $(2 \mu \mathrm{g} / \mathrm{mL})$ as a coating antigen was prepared in CBS (pH 9.6), purified ascites produced by NT-1 hybridoma was diluted 1:50000 in PBS ( $\mathrm{pH} 7.4$ ), NT was prepared in PBS containing $10 \%$ of methanol, GaMIgG-HRP was diluted $1: 1000$ in incubation buffer.

\subsection{Cross-reactivity of NT analogs}

Investigations on cross-reactivity of the antibody obtained are crucial for assessing the results. Therefore, a number of compounds, structurally related to NT, were tested with icELISA to characterize the properties of the antibody. Figure 5 shows all of the candidate compounds examined in this paper. Of all the cross-reacting steroids, $\alpha$-NT had the highest cross-reactivity data $(62 \%)$, but negligible cross- reactivity $(<0.01 \%)$ with the other steroids was observed.

\subsection{Effects of methanol in assay buffer}

NT is difficultly dissolved in water; therefore, suitable hydrotropic solvents are needed. Methanol is a commonly used agent for this, so it was used in this study. The effects were estimated by running standard curves under various conditions. The maximum absorbance ( $A_{\max }$, the absorbance value at zero concentration of NT) and half-maximum inhibition concentration ( $\mathrm{IC}_{50}$ values) were calculated, and the results are shown in Figure 6. Based on the results, 10\% methanol is suitable for this study.

\subsection{Matrix effects in urine samples}

It has been known that various substances in urine can affect antigen-antibody interaction in immunoassays. Dietary components, higher ionic strengths and the $\mathrm{pH}$ values strongly suppress the $\mathrm{IC}_{50}$ value and the maximum absorbance. When we tried to analyze urine samples without any pretreatment, spiked samples gave a very high background signal, resulting in an apparent absorbance decrease. A comparison between calibration plots for NT prepared in PBS and those prepared in different dilutions of urine gave clear evidence of a matrix effect (Figure 7). It was found that the 1:20 dilution in cattle urine gives inhibition curve that are almost the same as that of PBS.

\subsection{Correlation studies between icELISA and LC-MS/ MS analysis}

The objective was to compare the results obtained from ELISA with those from LC-MS/MS to determine the effectiveness of the icELISA method developed here. Therefore, cattle urine spiked samples were analyzed to obtain a direct comparison between the two methods. The results showed that the icELISA method gave a good correlation with the LC-MS/MS results $\left(R^{2}=0.9871\right.$, shown in Figure 8$)$, indicating the accuracy of the icELISA method developed here. Consequently, there was no risk of a false-positive result during the analysis of urine samples by the icELISA.

To further validate the immunoassay for detecting 19nortestosterone residues, authentic urine was assessed by collecting 15 samples from a local abattoir and subjecting them to icELISA and comparing the results with LCMS/MS analysis. The results showed that the residue levels ranged from 0.06 to $0.35 \mu \mathrm{g} / \mathrm{kg}(0.06-0.35 \mathrm{ng} / \mathrm{mL})$ for ELISA, compared with $0.05-0.32 \mu \mathrm{g} / \mathrm{kg}(0.05-0.32 \mathrm{ng} / \mathrm{mL})$ for LC-MS/MS. This means that the immunoassay developed here can be reliably used for real sample analysis.

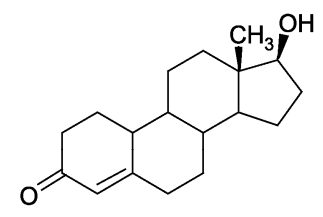

Nortestosterone (100\%)

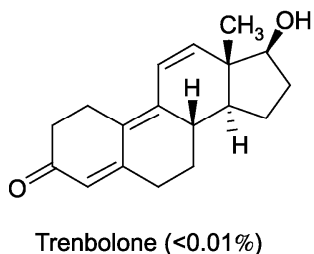

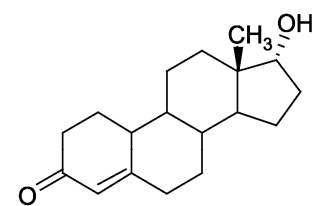

$\alpha$-Nortestosterone (62\%)

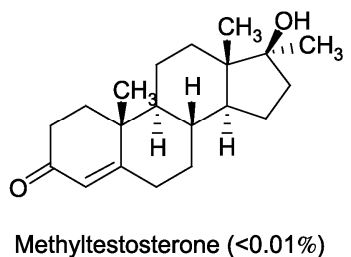<smiles>CC12CCC3C(CCC1OC2O)C1CCc2cc(O)ccc2C13</smiles>

Estradiol $(<0.01 \%)$<smiles>C[C@]12CCC[C@]3(C)CCC(=O)C(Cl)=C3[C@@H]1CC[C@@H]2O</smiles>

Clostebol $(<0.01 \%)$

Figure 5 Cross-reactivity of structurally related steroids in the NT immunoassay. 


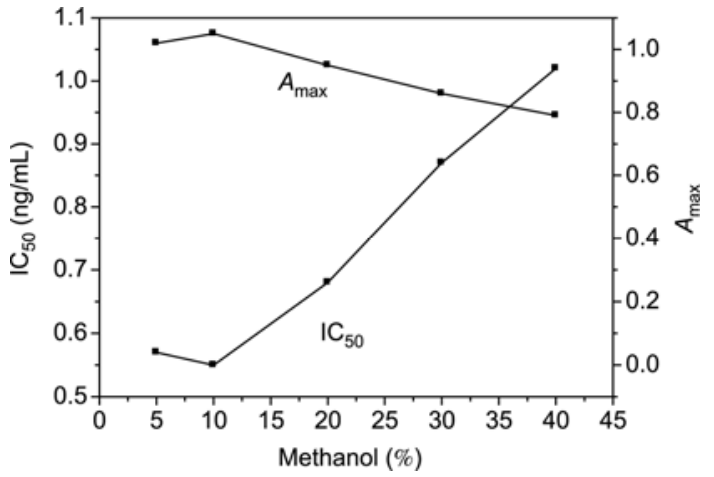

Figure 6 Effects of methanol concentrations on immunoassay. Each point represents the mean of three replicates.

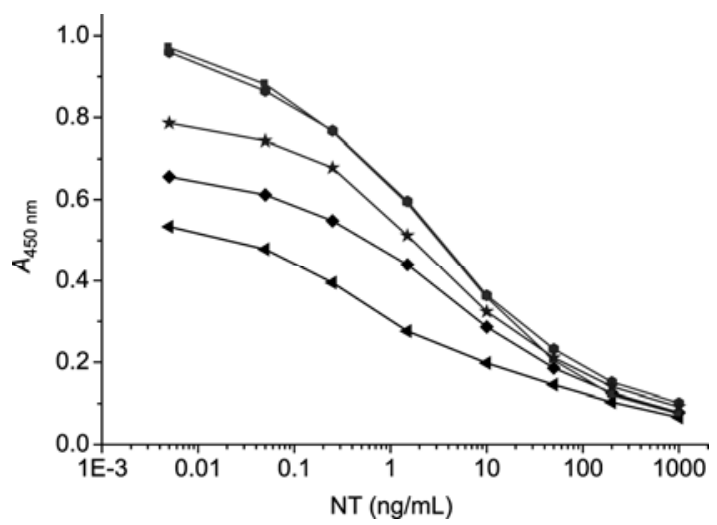

Figure 7 NT standard curves in the diluted urine samples. PBS (a), 2 -fold dilution $(\boldsymbol{\bullet})$, 5-fold dilution $(\bullet)$, 10-fold dilution $(\star)$, 20-fold dilution $(\bullet)$. Each point represents the average of three separate assays done in triplicate.

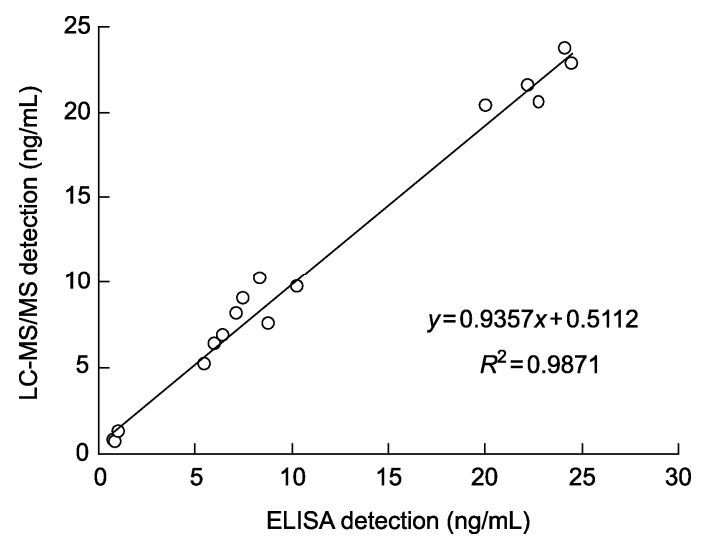

Figure 8 Correlation studies between ELISA detection and LC-MS/MS detection in cattle urine samples fortified with different concentrations of NT.

\section{Discussion}

Binding capacity, sensitivity, affinity and specificity determine the main immunity characteristics of an antibody, and immunoassays are based on the reciprocal conjugating activity between antigen and antibody. The polyclonal antibody $(\mathrm{pAb})$ usually contains unrelated or other antigenic immunoglobulin, as well as a mixture of products induced by different antigen determinants. Therefore, the quality differences in $\mathrm{pAb}$ causes difficulties for the immunoassay method. A homogeneous and unlimited supply of mAb became available following the development of the hybridoma technique by Köhler and Milstein [18]. Using this technology, we have screened five hybridoma cell lines in which two constant strains, named NT-1 and NT-2, had the highest titer $\left(1: 2.56 \times 10^{5}\right)$ and sensitivity $(0.55$ and $0.58 \mathrm{ng} / \mathrm{mL})$. Based on the NT-1 hybridoma, a heterologous icELISA standard curve has been developed for detecting NT residue in cattle urine.

The affinity constant $(\mathrm{Ka})$ of the antibody has an important effect on the quality of NT mAb. It demonstrates the conjugating ability between the antibody $(\mathrm{Ab})$ and hapten, or one antigen determinant, and is a significant signal of antibody stability. Thus it is important to be able to measure $K$ a. The ordinary methods include equilibrium dialysis, Scatchard plot, Batty saturation method, competitive binding, the precipitation method and other methods. The Batty saturation method used in this article is simple, rapid, and reliable. The use of serial dilutions of $\mathrm{Ab}$ resulted in a sigmoid curve of $A$ versus the logarithm of total $\mathrm{Ab}$ added to the well. The $A-50$ values for different antibodies were a reflection of the relative number of epitopes on the $\mathrm{Ag}$ that were identified by different antibody paratopes, provided excessive mAb was used. According to James [27], the five hybridomas all produced high-affinity antibodies (between $10^{7}$ and $10^{12} \mathrm{~L} / \mathrm{mol}$ ).

It is essential to produce highly molecular or structurally specific antibodies to NT hapten in the immunoassay. Heterologous icELISA was employed to judge the cross-reactivity of constant NT-1 mAb, in which the hapten-OVA conjugate used for plate coating was synthesized by a mixed-anhydride technique and the immunogen was prepared by employing a C-17 coupled steroid-protein conjugate through EDC method, thus overcoming unwanted crossreactivity (nonspecific binding) [28,29]. It is believed that the hapten- or antigen-antibody interaction is dependent on the spatial structure defined by molecular shape and geometry complementary bonding, and on low-energy interactions such as hydrogen bonding, hydrophobic interaction, and electrostatic or dipole-dipole forces. For our cross-reactivity data, the results were expected, considering that $\alpha-\mathrm{NT}$ and NT have very similar chemical structure while other analogs have an extra alkyl group, double bond or chlorine atom on the cyclopentano perhydro-phenanthrene, resulting in obvious steric hindrance.

This work was supported by the Eleventh Five-Year Plan for National Science and Technology of China (2006BAK02A21/1) and Henan Innovation Project for University Prominent Research Talents (2010HASTIT026).

1 Sterk S, Herbold H, Blokland M, et al. Nortestosterone: Endogenous in urine of goats, sheep and mares? Analyst, 1998, 123: 2633-2636 
2 Sauer M J, Samuels T P, Howells L G, et al. Residues and metabolism of 19-nortestosterone laurate in steers. Analyst, 1998, 123: 2653-2660

3 Conneely G, Aherne M, Lu H, et al. Development of an immunosensor for the detection of testosterone in bovine urine. Anal Chim Acta, 2007, 583: 153-160

4 Roda A, Manetta A C, Portanti O, et al. A rapid and sensitive 384-well microtitre format chemiluminescent enzyme immunoassay for 19-nortestosterone. Luminescence, 2003, 18: 72-78

5 Conneely G, Aherne M, Lu H, et al. Electrochemical immunosensors for the detection of 19-nortestosterone and methyltestosterone in bovine urine. Sens Actuators, 2007, 121: 103-112

6 Yamada M, Aramaki S, Kurosawa M, et al. Simultaneous doping analysis of main urinary metabolites of anabolic steroids in horse by ion-trap gas chromatography-tandem mass spectrometry. Anal Sci, 2008, 24: 1199-1204

7 Bahrke M S, Yesalis C E. Abuse of anabolic androgenic steroids and related substances in sport and exercise. Curr Opin Pharmacol, 2004, 4: 614-620

8 Baume N, Avois L, Schweizer C, et al. Nandrolone excretion in trained athletes: Interindividual variability in metabolism. Clin Chem, 2004, 50: 355-364

9 Akhter J, Hyder S, Ahmed M. Cerebrovascular accident associated with anabolic steroid use in a young man. Neurology, 1994, 44: 2405-2406

10 Brännvall K, Bogdanovic N, Korhonen L, et al. 19-Nortestosterone influences neural stem cell proliferation and neurogenesis in the rat brain. Eur J Neurosci, 2005, 21: 871-878

11 EC (1996) Council Directive (96/22/EC) of 29 April 1996 concerning the prohibition on the use in stock farming of certain substances, having a hormonal or thyrostatic action and of beta-agonists, and repealing Directives 81/602/EEC, 88/146/EEC and 88/299/EEC. Off J Eur Commun, L125, 3-9

12 Bagnati R, Fanelli R. Determination of 19-nortestosterone, testosterone and trenbolone by gas chromatography-negative-ion mass spectrometry after formation of the pentafluorobenzyl carboxymethoxime-trimethylsilyl derivatives. J Chromatogr, 1991, 547: 325-334

13 Seo J, Kim H Y, Chung B C, et al. Simultaneous determination of anabolic steroids and synthetic hormones in meat by freezing-lipid filtration, solid-phase extraction and gas chromatography-mass spectrometry. J Chromatogr A, 2005, 1067: 303-309

14 Dehennin L, Bonnaire Y, Plou P. Urinary excretion of 19-norandrosterone of endogenous origin in man: Quantitative analysis by gas chromatography-mass spectrometry. J Chromatogr B, 1999, 721: 301-307

15 Kim J Y, Choi M H, Kim S J, et al. Measurement of 19-nortestosterone and its esters in equine plasma by high-performance liquid chromatography with tandem mass spectrometry. Rapid Commun Mass Spectrom, 2000, 14: 1835-1840
16 Pozo O J, Van Eenoo P, Deventer K, et al. Development and validation of a qualitative screening method for the detection of exogenous anabolic steroids in urine by liquid chromatography-tandem mass spectrometry. Anal Bioanal Chem, 2007, 389: 1209-1224

17 Grace P B, Drake E C, Teale P, et al. Quantification of 19-nortestosterone sulphate and boldenone sulphate in urine from male horses using liquid chromatography/tandem mass spectrometry. Rapid Commun Mass Spectrom, 2008, 22: 2999-3007

18 Köhler G, Milstein C. Continuous cultures of fused cells secreting antibodies of predefined specificity. Nature, 1975, 256: 495-497

19 Chen Y, Wang Z, Wang Z, et al. Rapid enzyme-linked immunosorbent assay and colloidal gold immunoassay for kanamycin and tobramycin in swine tissues. J Agric Food Chem, 2008, 56: 2944-2952

20 Zhao S Q, Sun Y M, Zhang C Y, et al. Studies on purification of methamidophos monoclonal antibodies and comparative immunoactivity of purified antibodies. Biomed Environ Sci, 2003, 16: 119-125

21 Wang Z, Zhu Y, Ding S, et al. Development of a monoclonal antibody-based broad-specificity ELISA for fluoroquinolone antibiotics in foods and molecular modeling studies of cross-reactive compounds. Anal Chem, 2007, 79: 4471-4483

22 Wang Y C, Guo Z Q, Li Y Z, et al. Production and characterization of anti-estrone monoclonal antibody. Biomed Environ Sci, 2002, 15: 103-112

23 Wang L, Zhang Y, Gao X, et al. Determination of chloramphenicol residues in milk by enzyme-linked immunosorbent assay: Improvement by biotin-streptavidin-amplified system. J Agric Food Chem, 2010, 58: 3265-3270

24 Watanabe E, Eun H, Baba K, et al. Synthesis of haptens for development of antibodies to alkylphenols and evaluation and optimization of a selected antibody for ELISA development. J Agric Food Chem, 2005, 53: 7395-7403

25 Zhang Y, He F, Wan Y, et al. Generation of anti-trenbolone monoclonal antibody and establishment of an indirect competitive enzyme-linked immunosorbent assay for detection of trenbolone in animal tissues, feed and urine. Talanta, 2011, 83: 732-737

26 Strahm E, Saudan C, Sottas P E, et al. Direct detection and quantification of 19-norandrosterone sulfate in human urine by liquid chromatography-linear ion trap mass spectrometry. J Chromatogr B, 2007, 852: 491-496

27 James W G. Monoclonal Antibodies: Principles and Practice. New York: Academic Press, 1983. 142-147

28 Beier R C, Ripley L H, Young C R, et al. Production, characterization, and cross-reactivity studies of monoclonal antibodies against the coccidiostat nicarbazin. J Agric Food Chem, 2001, 49: 4542-4552

29 Liang Y, Liu X J, Liu Y, et al. Synthesis of three haptens for the class-specific immunoassay of O,O-dimethyl organophosphorus pesticides and effect of hapten heterology on immunoassay sensitivity. Anal Chim Acta, 2008, 615: 174-183

Open Access This article is distributed under the terms of the Creative Commons Attribution License which permits any use, distribution, and reproduction in any medium, provided the original author(s) and source are credited. 